Yutaka Ueno*, Shinya Muraoka and Tetsuo Sato

\title{
Skeletal animation for visualizing dynamic shapes of macromolecules
}

https://doi.org/10.1515/jib-2018-0098

Received April 2, 2019; accepted October 27, 2020; published online November 30, 2020

\begin{abstract}
We apply a skeletal animation technique developed for general computer graphics animation to display the dynamic shape of protein molecules. Polygon-based models for macromolecules such as atomic representations, surface models, and protein ribbon models are deformed by the motion of skeletal bones that provide coarse-grained descriptions of detailed computer graphics models. Using the animation software Blender, we developed methods to generate the skeletal bones for molecules. Our example of the superposition of normal modes demonstrates the thermal fluctuating motion obtained from normal mode analysis. The method is also applied to display the motions of protein molecules using trajectory coordinates of a molecular dynamics simulation. We found that a standard motion capture file was practical and useful for describing the motion of the molecule using available computer graphics tools.
\end{abstract}

Keywords: molecular dynamics; molecular visualization; normal mode analysis; structure of protein molecule.

\section{Introduction}

Recent computer graphics technology has advanced the high-resolution rendering of large-scale geometrical models with fine, smooth animation that is useful for molecular science. In fact, the consumer game industry has driven the development of leading software and tools for polygon-based models that support universal shape description. These graphical models can be used to describe hypothesized molecular mechanisms by means of animations. In a molecular 3D model of cell components, hundreds of molecules may be integrated into an animation using recent software. For example, ePMV [1] is a new-generation molecular graphics plugin that works as an add-on tool for general-purpose 3D computer graphics (CG) animation software such as Maya (Autodesk Inc.) [2] and Blender (Blender Foundation). On the other hand, molecular dynamics simulation results can be visualized by molecular graphics programs such as UCSF Chimera [3], Cn3D [4] or BioBlender [5] using atomic coordinates. However, it is not a trivial task to place many molecules in a graphics scene. Eventually many protein models depicted in a virtual cell Atlas could be animated to demonstrate proteinprotein interactions.

To accomplish this goal, we used polygon mesh models of a coarse-grained molecular surface, and displayed the dynamic conformational change of the molecules. We apply the skeletal animation method $[6,7]$ for molecular graphics to demonstrate the results of molecular simulation. The skeletal animation method takes advantage of recent advances in 3D CG hardware to render the shape changes of fine detailed polygon mesh models. It has been used in the media industry for CG animation films and game software development.

To apply motions to the polygon mesh models of molecular objects, we introduce skeletal bones whose movements deform the models. Figure 1 illustrates surface models of myosin made from atomic coordinates;

\footnotetext{
*Corresponding author: Yutaka Ueno, Artificial Intelligence Research Center, National Institute of Advanced Industrial Science and Technology, 2-4-7 Aomi, Koto-ku, Tokyo 135-0064, Japan, E-mail: uenoyt@ni.aist.go.jp. https://orcid.org/0000-0002-3124-2518 Shinya Muraoka, Graduate School of Information Science, Nara Institute of Science and Technology, Takayama 8916-5, Ikoma, Nara 630-0192, Japan, E-mail: shinya.muraoka@outlook.com

Tetsuo Sato, Department of Radiological Technology, Gunma Prefectural College of Health Sciences, 323-1 Kamioki, Maebashi, Gunma 371-0052, Japan, E-mail: satote@gchs.ac.jp
}

¿ Open Access. @ 2020 Yutaka Ueno et al., published by De Gruyter. $\Subset$ BY This work is licensed under the Creative Commons Attribution 4.0 International License. 


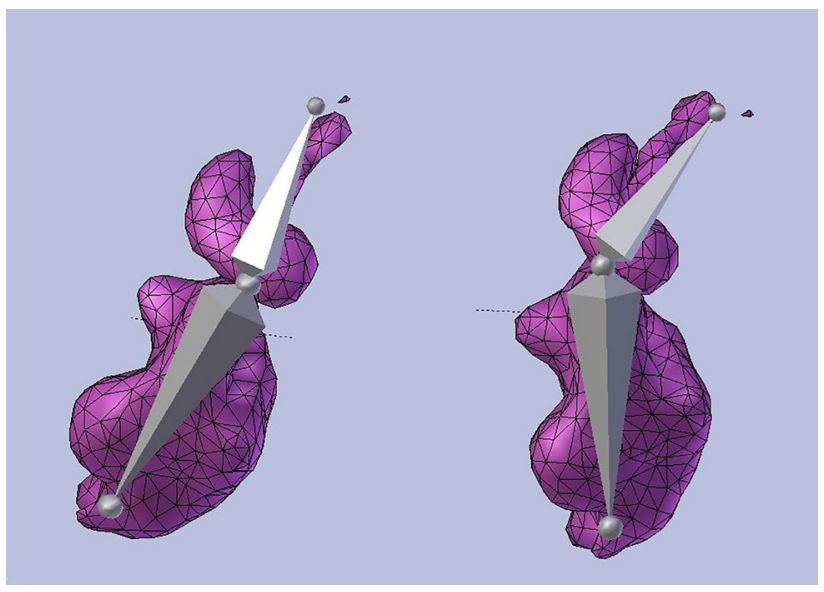

Figure 1: A molecular surface model of myosin (pdb 1MYS) deformed by skeletal animation. The hypothetical bending motion of the myosin, known as the power stroke, can be examined by defining the translation and rotation operations.

hypothetical movements of the molecule described in molecular biology textbooks are applied by means of skeletal bones. Bones can be superimposed on the polygon mesh models at arbitrary locations and with arbitrary movements. This ability is useful for describing conformational changes in, and the flexibility of, protein molecules. With typical 3D CG software, motions are described as sets of poses, and these sets are defined as key frames with translation and rotation at the specified time, and saved as animation scripts.

Our approach is to first describe coarse-grained motions for the normal mode analysis of proteins [8]. These calculations give the inherent flexibility of protein molecules and the spectroscopic and physical properties that are observed experimentally. Coarse-grained motion descriptions are useful because the motions are mostly described with groups of atoms based on protein domains.

In this study we demonstrate that skeletal animation is a practical tool for visualizing molecular simulation results at low computational cost. The method was used to place hundreds of molecules in a graphics scene of molecules in a cell. A motion capture file format supported by general-purpose animation production software is introduced to describe the motions of molecular skeletons. Although the file contains only a single motion at fixed length for making a movie, many existing software programs already support the file format for skeletal animation. We employed Blender, a 3D CG animation software package for movies that implements various functions for skeletal animation using the motion capture file format. With our results for molecular surfaces, protein ribbon models, and atomic models, we discuss possible performance improvements for the rendering of animated models using our method.

\section{Related works}

The skeletal animation method is also called rigging in the animation industry. The Rigging Kit for Molecular Maya (Digizyme Inc.) [9] was recently announced as an extension module to the animation construction software Maya. The method described in this study may also be applicable for work with Maya, although the cost of this commercial software is considerably high.

With the ePMV software suite, skeletal animation using alpha-carbon atoms with the inverse kinetics technique has been shown to support smooth handwork modeling. For example, morphing animation can demonstrate interactions between two different conformations of proteins in a PDB file, as well as in UCSF Chimera. Since the methods use the atomic coordinates described in the multiple model PDB file, increasing numbers of atom will drastically reduce the performance of the rendering software. A similar function is also available in Pyrite, a Blender plugin for molecular dynamics simulations [10].

Given an understanding of the molecular interaction of protein molecules, cellPACK [11] demonstrated large scale construction of the protein molecular world in the cell. Although simple rigid body translational motions showed realistic molecular behavior, the conformational changes to these molecules should also be 
demonstrated. Our study tries to provide such motions based on the normal mode analysis and molecular dynamics. Among recent studies of biological visualization, such as those discussed in the Baaden review article [12], one report that has generated intriguing discussion is a study using Blender to edit the molecular behaviors of membrane proteins based on microscopic observations [13].

\section{Methods}

Figure 2 contains a work-flow chart of our method for using the skeletal animation feature available in Blender. We implemented programs to define skeletal bones from the molecular structure and simulation data into files readable by Blender.

\subsection{The skeletal animation method}

A polygon mesh object is deformed by altering the vertex coordinates of the polygons. Once a skeleton consisting of a numbers of bones is assigned to the polygon model, each vertex can be transposed according to the movements of related bones. Each bone has two coordinates and an axis vector that is also defined as a rotation and translation matrix. All vertices $\vec{v}_{j}$ describing the mesh object are translated by different matrices of the near bones, and the mesh object deforms from its original structure. Among the different algorithms for mixing matrices, the simplest method is called "linear blend skinning". Using matrix $M_{j}$ for the $j$ th bone, the transformed coordinates of the vertices $\vec{v}_{j}$ are linearly mixed with those from several close bones [6]:

$$
\vec{v}_{i}^{\prime}=\sum_{j} w_{i, j} M_{j} \vec{v}_{i}
$$

Weights $w_{i, j}$ will be assigned to the ith vertices using the distance to the $j$ th bone. Blender implements this method to automatically calculate the weights, but then provides a graphical editor to modify the weights. When the deformed polygon mesh objects are not satisfactorily smooth, the weights can be modified manually using an interactive user interface. Figure 3 is an example of such a user interface implemented in Blender. It allows the vertices to be painted at a specified weight value.

For the skeletal animation, a standard file format called BVH was proposed by BioVision Inc [14]. First the hierarchical bones are defined with the top and bottom as the initial locations. A single sequence of motion data for the translations and rotations follows. Currently, various BVH data for human and other objects useful for movie media creation are available as both commercial and free data. We employed the coordinate values scaled to $10 \mathrm{~nm}$ in the 3D Blender scene, as most protein renderings fit a default CG scene.

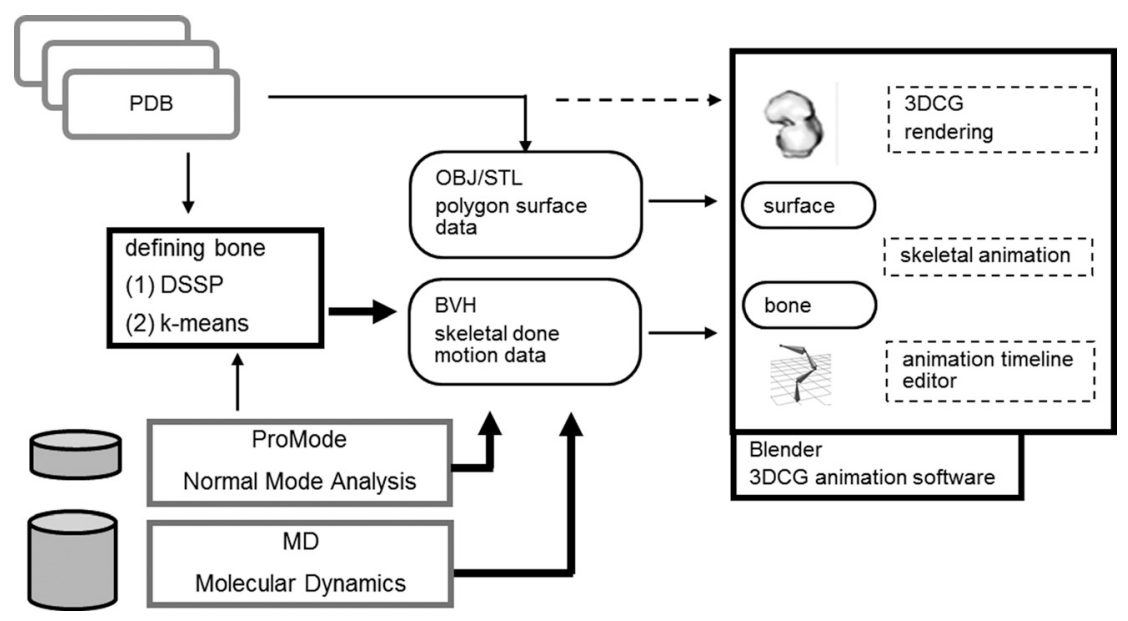

Figure 2: Work flow for the proposed skeletal animation using blender. Molecular objects are built from the atomic coordinate model PDB onto the polygon surface in the OBJ or STL format. They are animated in blender using skeletal animation according to the defined bones. The two methods used for defining bones are described in the text: (1) DSSP and (2) k-means. Results of normal mode analysis and molecular dynamics calculation are converted to the motion data saved in BVH file format to facilitate handling with existing 3D CG animation software. 


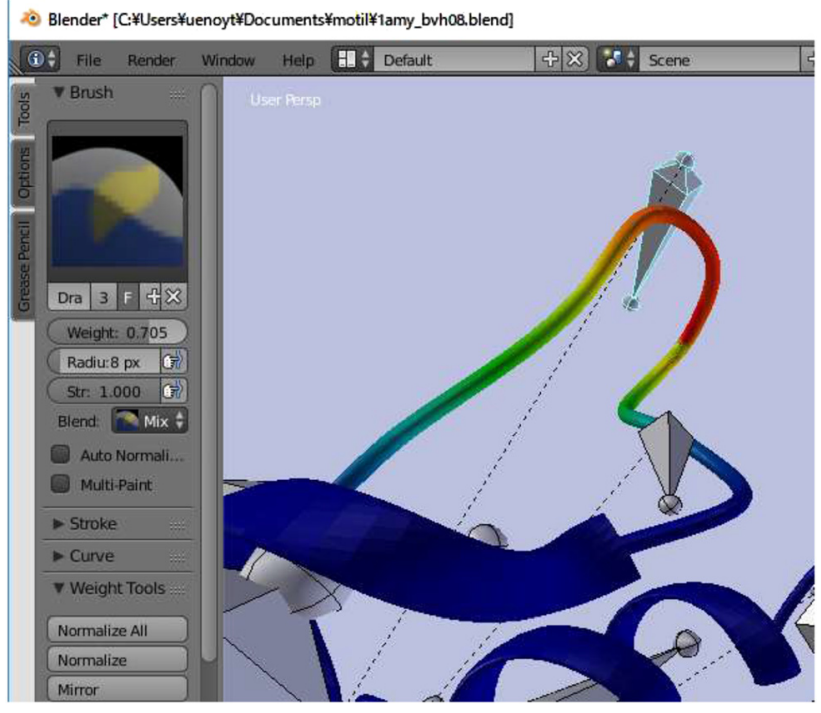

Figure 3: User interface provided by blender to adjust the bone weight for each polygon vertex. For the flexible tube representing a loop region of the peptide chain, vertices are selected and the "weight paint" mode allows bone-weight changes assigned to those vertices, where the weight value is chosen on the left color panel and painted using a mouse.

\subsection{Defining bones for the molecular object}

3.2.1 Bones from the atomic model: Bones for the molecular objects made from the atomic model are considered a coarse-grained model. In order such a model to yield natural movements, the normal mode analysis of thermal vibration based on molecular mechanics is used [15]. We tested a simple method by grouping atomic coordinates, including information from the major normal mode vectors. The atomic coordinates and major $\mathrm{m}$-sets of the normal mode vector provide a $3 \mathrm{~m}+3$ dimension vector for all atoms, and they are grouped by k-means clustering. With k-group center coordinates, the closest pairs give (k-1) bones, the axes of which are derived from the adjacent group center.

3.2.2 Bones by the secondary structure of protein: For protein molecules, the widely used ribbon model preserves an alpha helix by assigning a single bone. Moreover, the coarse-grained bone can also be derived from the secondary structure by DSSP analysis [16], where successive secondary structure residues are assigned as a single bone. Small bones, except for helix and sheet bones, can be merged; typically, six residues represent the minimum size. For the $\alpha$-helix, the three starting and three ending alpha carbon positions are averaged to create the two-bone coordinates. The axis vector was derived from the alpha carbon position of the fourth starting residue.

\subsection{The superposition of normal mode motions}

While the normal mode vibration analysis provides the major components of physical object motion, an arbitrarily linear superposition is allowed to represent or reconstruct the practical motion of the object using coefficient $A_{m}$ and phase shift $p_{m}$ :

$$
\overrightarrow{W_{j}}(t)=\sum_{m}^{\text {mode }} \vec{v}_{m j} \cdot A_{m} \cdot \sin \left(2 \pi \mathrm{w}_{m} t+p_{m}\right)
$$

where, $v_{m j}$ is the $m$ th vibration mode for the atom, and $w_{m}$ is the vibration frequency. While the individual motion provides direct evidence of the object's flexibility, in practice the motion should always be mixed. The obtained motion $W j(t)$ indicates displacement vectors to the atomic coordinates to yield the animated motion of the atomic model. We used all 10 of the lowest frequency normal modes providing vibration frequencies that dominate major dynamic motions of the protein available at the ProMode Elastic server, following their calculation methods [15]. Changing the phase gives different aspects of motions that may appear some time later in other compositions. There are also different calculation servers for the normal mode analysis, such as ElNemo [17], which calculates more than 10 normal modes for further analysis about the important number of normal modes.

\subsection{Assigning bones to a model in blender}

Our method is not limited to use in Blender, but may also be applied in other 3D CG animation software such as Maya, Cinema4D (MAXON Computer GmbH), and 3ds Max (Autodesk Inc.), as long as the BVH file format is supported. Here, we describe a typical 
operation in Blender. The polygon mesh models of the molecular model were saved in files by the molecular graphics package, UCSF Chimera. Typically, the OBJ format contains color information that is useful for color models by atomic elements or chemical properties. For protein ribbon models or a surface model, the STL format is much more compact. After loading the polygon model, a corresponding bone file in BVH format is imported to Blender. Then, the "parent" command will assign the bone to the target polygon mesh, where the "automatic weight" option is selected for the skeletal animation.

Creating animation using Blender requires a lot of knowledge and experience due to the complexity of the workflow required to make movies using 3D polygon objects. Even with an improved graphical user interface and a variety of use case materials on the Internet, Blender has not been widely utilized in scientific research. Therefore, we concluded that the animation script file should be shared in order to ensure the reproducibility of the visualization method. We have identified useful tutorial materials to help scientific researchers use Blender; for example, the BioBlender website [5], and CellBlender in MCell website [18] with useful documentation. There is a modeling study for lipid bilayers that demonstrates the advanced use of Blender available on its website [19].

\section{Applications}

\subsection{Thermal vibration of proteins by normal mode analysis}

The Promode-Elastic server provides the results of a normal mode analysis for the PDB protein atomic model with graphical visualization for the individual vibration mode. For an alternative visualization of the vibration mode, we tried the arbitrary superposition of several normal modes to generate possible thermal fluctuations of the molecule. After visual inspections, the amplitude $A_{m}$ was set to 2 and the phase shift $p_{m}$ was 0 for all modes. Changing these parameters does not really affect the visualization of the overall non-periodic fluctuation.

Figure 4 is a ribbon model for $\alpha$-amylase 1AMY in PDB with 37 overlapping skeletal bones. Figure 5 is a snapshot with three superimposed conformations of the animated model showing that the overall shape deformation of the protein with 4,088 atoms is well described by the skeletal animation. In particular, mobile
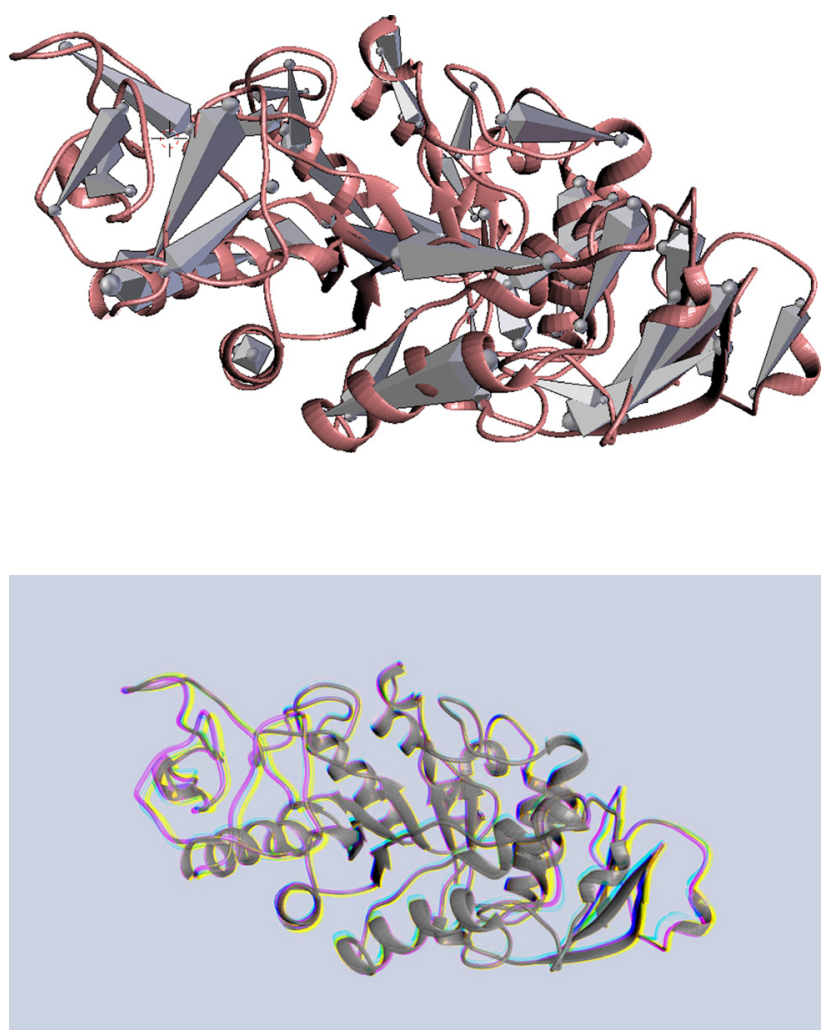

Figure 4: A sample skeleton introduced for $\alpha$-amylase (pdb 1AMY). These bones represent motions converted for the normal mode analysis, and the molecular dynamics trajectory is demonstrated on our web page [20]. In the loop regions, bone motions frequently become larger than those in the other regions. In that case, smaller bones should be used for smoother animation.

Figure 5: A snapshot of the animation display for $\alpha$-amylase (pdb 1AMY). Three conformations at different time frames, depicted in yellow, cyan, and magenta, are simply superimposed to demonstrate the movement. Overlapped regions then become gray. 
regions consisting of loop regions were well distinguished compared to more rigid portions, including alphahelix or beta-sheet components. The normal mode frequency was adjusted to make 0.02 radian sinusoidal steps for a $1 \mathrm{~cm}^{-1}$ wave number, which is saved in a standard movie sequence with 24 frames per second (fps). Sample movies and scripts are available from our website [20] with supplemental materials describing the Blender script.

We also examined the discrepancy between the animated trajectory coordinates and the reference normal mode displacement. The animated coordinates of the atomic model were retrieved by a script in Blender for the lower 10 normal mode movements, and the root mean square difference of the animated coordinates was $0.004-0.012 \mathrm{~nm}$ when the amplitude was unity.

\subsection{Coarse-grained trajectories of molecular dynamics simulations}

In order to provide a quick preview of the molecular dynamics trajectory, we applied our method for the coarse-grained trajectory of atomic movements. Once the skeletal bones are defined for the initial coordinates, the molecular dynamics trajectory can be approximated by the motion of the skeletal bones. The skeletal bones do not require accuracy in terms of grouping atoms; the subsequent motion data assigned to the groups already contains the mean positions of atoms. The example molecule discussed above, $\alpha$-amylase, was also used in a simple molecular dynamics simulation in an aqueous environment. GROMACS (ver 5.0) was used with the GROMOS96 (43a1) united-atom force field on an Ubuntu Linux, 32 GBytes main memory, 8-thread Core i7-4765t processor. Based on a standard protocol for the constant number of particles, pressure, temperature (NPT) ensemble, duration 8 ps dynamics at $300 \mathrm{~K}$ in atmospheric pressure, 4,000 frames at a $2 \mathrm{fps}$ time step were calculated. Trajectory coordinates are saved at every 10th frame, resulting in a total of 400 trajectories of 4,088 atoms, generating a PDB file 8 MBytes in size. Then a BVH motion capture file of 704 KBytes was generated for the same skeleton with 37 bones, as in the previous normal mode analysis.

Skeletal animation runs on a conventional personal computer with low computational cost. For example, a molecular dynamics trajectory visualization using an atomic model of $\alpha$-amylase like that in Figure 5 consumes an additional $42 \mathrm{MB}$ of main memory, while the molecular dynamics simulation trajectory requires a viewer program. UCSF Chimera (ver 1.10.1), for example, required an additional $350 \mathrm{MB}$ of main memory.

\subsection{Motion parts for making scenes of the molecular world}

Our main goal in this motion description method is to animate the protein molecules used in constructing scenes of the molecular world in a cell. Examples of the script file available from the website [21] demonstrate some of the basic biochemistry textbook contents, including: the model of motor protein myosin and actin extended to the skeletal muscle filament; the tread milling motion of the actin filament; and an in vitro motility assay for the movements of microtubules on kinesin proteins. Our work constructing a hypothetical animation for the starch hydrolysis of $\alpha$-amylase with flexible molecular motion is described in the supplemental material.

In addition to the globular molecular shape changes, we also demonstrated an animated atomic model of the active site of $\alpha$-amylase reported by EzCatDB, a database for enzyme catalytic mechanisms [22]. Using several atomic models of the active site investigated by X-ray crystallography, interpolated atomic coordinates between several conformations were generated by UCSF Chimera. Currently, this animation is a series of atomic coordinate models that we were not able to describe by skeletal bones. Figure 6 shows an example of the handwork illustrations for atomic models in the chemical context. 


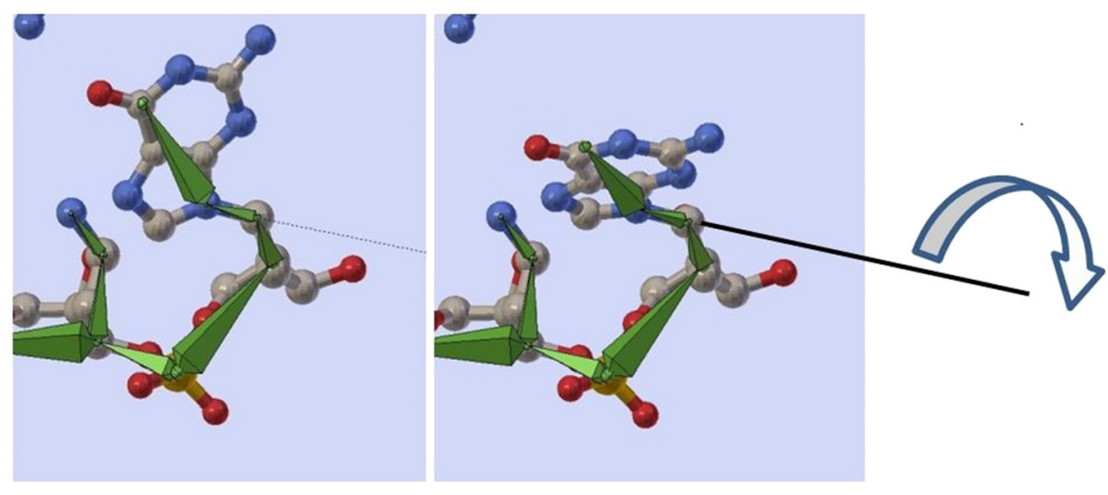

Figure 6: A possible skeletal animation for the atomic model of guanine. The groups of atoms for macromolecules may be defined and moved by bones to provide intuitive modeling such as dihedral rotations, which we plan to address in future work.

\section{Discussions}

\subsection{A superposition of normal mode}

The normal mode movements of a globular molecule have been visualized using illustrations of individual components, where the corresponding frequency provides the spectroscopic property of the molecule. We attempted to visualize these modes by combining major normal modes. The movement looks very similar to a thermal fluctuation, and is thus a reasonably demonstrated mobile molecular segment. There are also different superposition coefficients that would make it possible to distinguish specific movements of the normal mode vibration. In comparisons with the molecular dynamics trajectory, the fast motion components are mostly smoothed out and only global shape changes give a different impression. Usually detailed motions of the molecular dynamics tend to differentiate into random motions; the normal mode fluctuations show unchanging characteristic views of the movements. These fluctuations would be preferable for the generalpurpose scientific visualization.

\subsection{Coarse-grained molecular dynamics trajectory}

The increasing data size obtained from large-scale molecular dynamics simulations yields huge amounts of data on the dynamics trajectories of macromolecules. In particular, even with a united-atom force field to reduce hydrogen atoms as in our example, protein complex structures are extremely large. Efficient methods to compress these trajectory data have been reported [23, 24], supporting the accurate conservation of simulation results. While these algorithms ensure a certain precision, our method may also be employed to visualize overall shape changes and domain motions where detailed atomic coordinates have an insignificant visual effect.

Additional bones may also be used for detailed rotational motions of protein side chains. When large changes in the atomic structure of the trajectory data exist, the automatic generation of such detailed bones would be possible.

\subsection{Improving the performance of recent graphics hardware}

The skeletal animation method could be improved by using a vertex buffer with recent graphics hardware that allows rapid change of vertices for rendered polygons. The hardware vertex buffer technique is supported by the OpenGL standard as an extension of functionality, and is further reinforced by other graphics libraries: DirectX (Microsoft), Vulkan (Khronos Group), or Metal (Apple). Because implementations of skeletal animation using graphics hardware are increasing in various application domains, molecular graphics software tools 
would also benefit from this technology. We began implementing our own rendering code with the vertex buffer in our original viewer program [25] to take advantage of graphics hardware for skeletal animation.

\subsection{Understanding protein motions and scientific visualizations}

We found that the BVH file format is useful for describing the motions of protein molecules using 3D CG tools with skeletal animation. Our future work will employ this molecular animation method for EzCatDB, a database for enzyme catalytic mechanisms [22]. In addition, various web-based bioinformatics tools may benefit from this method for displaying animated proteins.

In scientific visualization studies, molecular mechanisms can be demonstrated by movies of proteins at work [12]. It is important for those movies to present the natural motions of molecules that cannot be captured by the experimental method. Skeletal animation is useful for describing the conformational change of a protein based on a hypothesis deduced from the structural biology literature. Such shape changes of molecular surface models have been studied previously [26]; however, they involved complex geometry calculations. When handworks attempt to illustrate arbitral deformations, the skeletal bone of the coarse-grained model is useful. Our method will also support such moving parts as the skeletal bones saved in the BVH format. Notably, it runs with very little computational cost, so it is possible to integrate many molecular complexes on the 3D CG scene. Meanwhile, BVH is a standard file format that is widely supported by a number of existing graphics software suites for 3D animation.

\section{Conclusions}

While scientific visualizations can now include portrayal of the motions of proteins at work, we studied a practical way to visualize the molecular simulation results of the thermal vibration on general-purpose 3D CG software. In addition to the hypothetical protein motions proposed to understand a protein's function, it is important for such movies to present the natural motions of molecules, which cannot be adequately captured by the experimental method. Our method of using the motion capture file format for protein motion can be used with modern computer graphics software tools that are fully supported by recent graphics hardware. Source code and animation scripts for this study are available on our website [20] at https://github.com/uenoyt/ skelmol.

Acknowledgments: We thank Prof. Shigehiko Kanaya and Dr. Naoaki Ono at the Nara Institute of Science and Technology for their useful discussions and mentoring in this study.

Author contribution: Ueno Y.: Conceptualization, Methodology, Writing. Muraoka S.: Software implementation, Data analysis, Visualization. Sato T.: Administration, Validation, Reviewing and editing.

Research funding: This work was supported by a Grant-in-Aid for Scientific Research on the Innovative Area "Molecular Robotics" (No. 24104004) from The Ministry of Education, Culture, Sports, Science, and Technology, Japan.

Conflict of interest statement: The authors state that they have no conflict of interest. All authors have read the journal's publication ethics and publication malpractice statement available at the journal's website and hereby confirm that they comply with all portions of the statement applicable to the present scientific work.

\section{References}

1. Johnson GT, Autin L, Goodsell DS, Sanner MF, Olson AJ. ePMV embeds molecular modeling into professional animation software environments. Structure 2011;19:293-303. 
2. Parent R. Computer animation complete: all-in-one learns motion capture, characteristic, point-based, and Maya winning techniques. San Francisco, CA, USA: Morgan Kaufmann Publishers Inc.; 2009.

3. Pettersen EF, Goddard TD, Huang CC, Couch GS, Greenblatt DM, Meng EC, et al. UCSF chimera-a visualization system for exploratory research and analysis. J Comput Chem 2004;25:1605-12.

4. Wang Y, Geer LY, Chappey C, Kans JA, Bryant SH. Cn3D: sequence and structure views for Entrez. Trends Biochem Sci 2000;25: 300-2. 4. R.M.

5. Andrei RM, Callieri M, Zini MF, Loni T, Maraziti G, Pan MC, et al. Intuitive representation of surface properties of biomolecules using bioblender. BMC Bioinf 2012;13(4 Suppl):S16. Available from: http://www.bioblender.org.

6. Kavan L. Direct skinning methods and deformation primitives. In: Skinning: real-time shape deformation, couses note. Singapore: ACM SIGGRAPH; 2014, vol. 2014. Available from: https://skinning.org/direct-methods.pdf.

7. Lander J. Skin them bones: game programming for the web generation. Game Dev 1998;5:11-16.

8. Wako H, Endo S. Normal mode analysis based on an elastic network model for biomolecules in the protein data bank, which uses dihedral angles as independent variables. Comput Biol Chem 2013;44:22-30. https://doi.org/10.1016/j.compbiolchem. 2013.02.006 A.

9. McGill G. Introduction to molecular Maya's rigging Kit. Available from: https://clarafi.com/training/introduction-to-molecularmaya-rigging-kit/.

10. Rajendiran N, Durrant JD. Pyrite: a blender plugin for visualizing molecular dynamics simulations using industry-standard rendering techniques. J Comput Chem 2018;5:748-55. 12.

11. Johnson GT, Autin L, Al-Alusi M, Goodsell DS, Sanner MF, Olson AJ. CellPACK: a virtual mesoscope to model and visualize structural systems biology. Nat Method 2015;12:85-91.

12. Baaden M. Visualizing biological membrane organization and dynamics. J Mol Biol 2019;431:1889-919.

13. Biere N, Ghaffar M, Doebbe A, Jäger D, Rothe N, Friedrich BM, et al. Heuristic modeling and 3D stereoscopic visualization of a Chlamydomonas reinhardtii cell. J Integr Bioinf 2018;15:20180003.

14. Meredith M, Maddock S. Motion capture file formats explained. Tech Rep Dept Comput Sci Univ Sheffield; 2001:CS-01-11 p. Available from: http://www.dcs.shef.ac.uk/intranet/research/public/resmes/CS0111.pdf.

15. Wako H, Kato M, Endo S. ProMode: a database of normal mode analyses on protein molecules with a full-atom model. Bioinformatics 2004;20:2035-43. Available from: https://pdbj.org/promode-elastic.

16. Kabsch W, Sander C. Dictionary of protein secondary structure: pattern recognition of hydrogen-bonded and geometrical features. Biopolymers 1983;22:2577-637.

17. Suhre K, Sanejouand YH. EINemo: a normal mode web server for protein movement analysis and the generation of templates for molecular replacement. Nucleic Acids Res 2004;32:W610-4.

18. M Cell and Cell Blender. Available from: http://mcell.org.

19. Mehmood G, Niklas B, Daniel J, Karsten K, Falk S, Olaf K, et al. 3D modelling and visualisation of heterogeneous cell membranes in blender. In: Proceedings of the 11th international symposium on visual information communication and interaction. New York, NY: ACM Press; 2018:64-71. Available from: http://cellmicrocosmos.org.

20. Skeletal animation for molecular models. Available from: https://github.com/uenoyt/skelmol.

21. Blender for molecular biology. Available from: https://github.com/uenoyt/blendmb.

22. Nagano N, Nakayama N, Ikeda K, Fukuie M, Yokota K, Doi T, et al. EzCatDB: the enzyme reaction database, 2015 update. Nucleic Acids Res 2015;43:D453-458. Available from: http://ezcatdb.cbrc.jp.

23. Huwald J, Richter S, Ibrahim B, Dittrich P. Compressing molecular dynamics trajectories: breaking the one-bit-per-sample barrier. J Comput Chem;37:1897-906. https://doi.org/10.1002/jcc.24405.

24. Spångberg D, Larsson DSD, van der Spoel D. Trajectory NG: portable, compressed, general molecular dynamics trajectories. J Mol Model 2011;17:2669-85.

25. Ueno Y, Asai K. A high-throughput graphics library designed for a portable molecular structure viewer. In: Altman R, editor. Pac symp biocomput. Singapore: World Scientific Press; 1998:201-12 p.

26. Eyal E, Halperin D. Dynamic maintenance of molecular surfaces under conformational changes. In: Proceedings of the 21st annual symposium on computational geometry. SCG '05. ACM, New York, NY, USA; 2005:45-54. https://doi.org/10.1145/ 1064092.1064102.

Supplementary Material: The online version of this article offers supplementary material (https://doi.org/10.1515/jib-2018-0098). 